\title{
AVALIAÇÃO DE MÉTODOS DE ESTIMATIVA DA EVAPOTRANSPIRAÇÃO DE REFERÊNCIA PARA TRÊS LOCALIDADES NO NORTE DE MINAS GERAIS
}

\author{
Leonidas Pena de Alencar ${ }^{1}$, Gilberto Chohaku Sediyama ${ }^{2}$, Henderson S. Wanderley ${ }^{3}$, Thomé Simpliciano Almeida ${ }^{4}$, \\ Rafael Coll Delgado ${ }^{5}$
}

\section{RESUMO}

No presente trabalho objetivou-se realizar o estudo comparativo dos métodos de estimativa da evapotranspiração de referência de Blaney Criddle, Hargreaves-Samani, Priestley-Taylor e Radiação com o método padrão PenmanMonteith (FAO 56) para as condições climáticas dos municípios de Montes Claros, Espinosa e Salinas, localizados no Norte de Minas Gerais. Foi utilizado um conjunto de dados diários de 10 anos (1996 a 2005) das temperaturas máximas e mínimas, velocidade do vento, insolação e umidade relativa do ar. Para comparar os valores de ETo estimados por meio das equações empíricas com os do método padrão universal de Penman-Monteith (FAO 56), foram considerados os parâmetros a e b da equação de regressão, o coeficiente de determinação $\left(\mathrm{R}^{2}\right)$ e de correlação de Pearson (r), o erro absoluto médio (EAM), a raiz do erro quadrático médio (REQM), o índice de concordância de Willmott(d) e o índice de confiança ou desempenho (c). Os métodos foram avaliados para duas estações do ano, uma de baixa demanda evaporativa para os meses de abril a setembro e outra de alta demanda entre outubro e março. Em ambos os casos, os métodos de Blaney Criddle e o de Priestley Taylor apresentaram os melhores resultados na estimativa da ETo. O método de Hargreaves-Samani apresentou o pior desempenho para as condições climáticas estudadas.

Palavras-chave: Blaney Cridlle, Hargreaves-Samani, Penman-Monteith, Priestley-Taylor, modelos empíricos, radiação.

\section{ABSTRACT \\ EVALUATION OF METHODS TO ESTIMATE EVAPOTRANSPIRATION AT THREE LOCATIONS OF NORTHERN MINAS GERAIS}

The objective of this study was to compare the methods of Blaney Criddle, Hargreaves-Samani, Priestley-Taylor and Radiation with the standard method Penman-Monteith (FAO 56), to estimate evapotranspirtaion under the climatic conditions of Montes Claros, Espinosa and Salinas, located in the northern Minas Gerais. Analysis was done using 10 years (1996 to 2005) daily data regarding maximum and minimum temperatures, wind speed, sunshine and relative humidity. To compare the ETo estimated by using empirical equations with the standard method of Penman-Monteith (FAO 56) the following parameters were used: ' $a$ ' and ' $b$ ' of the regression equation, coefficient of determination $\left(\mathrm{R}^{2}\right)$, correlation coefficient (r), the mean absolute error, root mean square error, the agreement index and the confidence or performance index. The methods were evaluated for two seasons, during low evaporative demand in the months of April to September, and high demand during October to March. In both seasons, the methods of Blaney and Criddle Priestley Taylor proved to be more precise. The method of Hargreaves-Samani was unsatisfactory under the weather conditions studied.

Keywords: Blaney Cridlle, Hargreaves-Samani, Penman-Monteith, Priestley-Taylor, empirical models, radiation.

\section{Recebido para publicação em 25/10/2010. Aprovado em 25/04/2011.}

1- Doutorando em Engenharia Agrícola/UFV, Viçosa, MG, Bolsista do CNPq, e-mail: lpaufv@hotmail.com

2- Engenheiro Agrônomo, Prof. Titular da UFV, DEA, Viçosa, MG, e-mail: g.sediyama@ufv.br

3- Meteorologista, Doutorando em Meteorologia Agrícola UFV, DEA, Viçosa, MG, e-mail: hendynho@hotmail.com

4- Físico, Doutorando em Meteorologia Agrícola UFV, DEA, Viçosa, MG, e-mail: thomeaj@hotmail.com

5- Meteorologista, Doutor em Meteorologia Agrícola, e-mail: rafaelcolldelgado32@gmail.com 


\section{INTRODUÇÃO}

Um dos requisitos fundamentais para estimar a quantidade de água necessária para uma produção agrícola ideal é entender efetivamente as relações entre as condições climáticas e a evapotranspiração (ET). A evapotranspiração é definida como a combinação de dois processos distintos, a evaporação da água diretamente da superfície do solo e a transpiração através dos estômatos das plantas (ALLEN et al., 2006).

Estimativas precisas da evapotranspiração são essenciais para identificar as variações temporais sobre a necessidade de irrigação, melhorar a alocação dos recursos hídricos e avaliar o efeito do uso da terra e mudanças na gestão do balanço hídrico (ORTEGA-FARIAS, et al., 2009). A quantificação da ET é um componente básico para a concepção, exploração e gestão dos sistemas de irrigação, sendo o principal responsável pela perda de água nas bacias hidrográficas.

Como tal, a evapotranspiração exerce uma grande influência sobre os processos hidrológicos, estando este componente estreitamente relacionado à dinâmica da umidade do solo, à recarga dos aquíferos e ao escoamento superficial (LIANG et al., 2008).

A evapotranspiração de referência (ETo) é definida como a taxa de evapotranspiração de uma superfície de referência, coberta por grama hipotética com altura de $0,12 \mathrm{~m}$, uma resistência de superfície de $70 \mathrm{~s} . \mathrm{m}^{-1}$ e um albedo de 0,23, com altura uniforme, sem restrições de água, crescendo ativamente e sombreando completamente o solo (ALLEN et al., 2006). O conceito de ETo foi originalmente introduzido para estudar a demanda evaporativa da atmosfera independentemente do tipo de cultura, fenologia e tratos culturais, sendo portanto, um termo mais usado em estudos climáticos.

Sabendo que a medição direta da evapotranspiração é uma tarefa difícil, o desenvolvimento de modelos hidrometeorológicos para avaliar a evapotranspiração resultou em contribuições importantes para o manejo da irrigação em escala global, regional e local (MAEDA et al., 2010). Muitas das equações empregadas na estimativa da ETo são empíricas, limitando seu uso a locais e climas similares àqueles onde foram efetuadas as suas determinações.

Durante as últimas décadas, foi desenvolvida uma grande variedade de métodos para a estimativa da ETo. Esses métodos são normalmente baseados em princípios físicos, equações empíricas ou mesmo em uma combinação de métodos físicos e empíricos. Os métodos empíricos são fundamentados em observações e análise estatística e geralmente são adequados para uma condição climática ou região específica (FOOLADMAND; AHMADI, 2009; e AHMADI; FOOLADMAND, 2008; GAVILÁN et al., 2006).

O método de Penman-Monteith (FAO 56) foi o método recomendado pela FAO como padrão para calcular ETo e tem sido utilizado em todo o mundo. No entanto, este método exige medições de vários elementos climáticos como temperatura do ar, umidade relativa, radiação solar e velocidade do vento.

Assim, a necessidade de vários elementos climáticos leva ao desenvolvimento de abordagens mais simples para estimar ETo. Neste contexto, vários métodos de estimativa de ETo têm sido relatados na literatura, alguns deles baseados em uma única variável climática - a radiação solar (BLANEY; CRIDDLE, 1950), (MAKKINK, 1957) e (PRIESTLEY; TAYLOR, 1972) ou a temperatura (HARGREAVES; SAMANI, 1985).

Em razão do grande número de métodos existentes para estimativa da ETo, a escolha do método mais adequado depende da disponibilidade de dados climatológicos, do nível de precisão exigido, da finalidade, se manejo da irrigação ou pesquisa, bem como do custo de aquisição de equipamentos (TAGLIAFERRE et al., 2010).

O conhecimento da evapotranspiração é de grande importância para a região Norte de Minas, visto que a região é uma das grandes produtoras de frutas em função dos perímetros irrigados implantados pela Codevasf e pelo DNOCS. Assim, o conhecimento da evapotranspiração de referência (ETo) é de extrema importância para o planejamento dos recursos hídricos e para a agricultura irrigada na região.

Vários estudos comparando os métodos de evapotranspiração são encontrados na literatura para diferentes regiões (OLIVEIRA et al., 2008; 
TAGLIAFERRE et al., 2010), porém os resultados variam muito entre os estudos, pois cada região apresenta características climáticas peculiares, o que dificulta ao usuário decidir sobre a conveniência de qual método adotar.

Assim, objetivou-se no presente estudo, comparar os métodos de estimativa da evapotranspiração de referência baseados na Radiação solar (FAO 24), Blaney Criddle (FAO 24), Priestley e Taylor (1972) e Hargreaves-Samani (1985) com o método padrão de Penman-Monteith (FAO 56), utilizando diferentes índices estatístico.

\section{MATERIAL E MÉTODOS}

A área de estudo abrange os municípios de Montes Claros (Latitude $14^{\circ} 44^{\prime}$ S, Longitude $43^{\circ} 52^{\prime}$ W, Altitude de $648 \mathrm{~m}$ ), Salinas (Latitude $16^{\circ} 10^{\prime} \mathrm{S}$, Longitude $42^{\circ} 17^{\prime} \mathrm{W}$, Altitude de $471 \mathrm{~m}$ ) e Espinosa (Latitude $14^{\circ} 55^{\prime} \mathrm{S}$, Longitude $42^{\circ} 49^{\prime} \mathrm{W}$, Altitude de $570 \mathrm{~m}$ ), localizadas na região Norte de Minas Gerais. Foram utilizados dados diários de temperatura máxima e mínima, umidade relativa do ar, velocidade do vento e insolação coletados nas estações climatológicas pertencentes ao Instituto Nacional de Meteorologia (INMET). O período de abrangência dos dados foi de 1996 a 2005, totalizando 10 anos de dados.

A estimativa da evapotranspiração de referência diária (ETo) foi obtida pelos modelos de PenmanMonteith, parametrizado pela FAO, Radiação, Blaney Criddle, Hargreaves-Samani e Priestley-Taylor.

\section{Método de Blaney Criddle (FAO 24)}

Com o objetivo de definir melhor os efeitos do clima nas necessidades hídricas das culturas, o método original de Blaney-Criddle (BLANEY; CRIDDLE, 1950), foi modificado por Doorenbos e Pruitt (1977) para obtenção da ETo, considerando os níveis gerais de umidade relativa mínima, velocidade do vento e insolação. $\mathrm{O}$ método de Blaney-Criddle modificado pela FAO é o seguinte:

$E T=a+b f$

em que

$a$ e b = são os coeficientes da equação linear que relacionam $\mathrm{f}$ e a ETo; e

$\mathrm{f}=$ é o fator da equação de Blaney Criddle dado pela equação:

$f=\mathrm{p}(0,46 T+8,13)$

em que

$\mathrm{p}=$ diária média anual de horas de brilho solar (\%); e $\mathrm{T}=$ é a temperatura do ar média $\left({ }^{\circ} \mathrm{C}\right)$.

As expressões propostas por Frevert et al. (1983) são utilizadas para o cálculo dos coeficientes de a e b.

$$
\begin{aligned}
& a=0,0043 \mathrm{UR}_{\text {MIN }}-\left(\frac{n}{N}\right)-1,41 \\
& b=\mathrm{a}_{0}+\mathrm{a}_{1} \mathrm{UR}_{\text {MIN }}+\mathrm{a}_{2} \frac{n}{N}+\mathrm{a}_{3} U_{d}+c \\
& c=\mathrm{a}_{4}\left(\frac{n}{N}\right) \mathrm{UR}_{\text {MIN }}+\mathrm{a}_{5} U_{d} \mathrm{UR}_{\text {MIN }}
\end{aligned}
$$

em que

$a_{o}=0,81917 ; a_{1}-(-0,0040922)$

$\mathrm{a}_{2}=1,0705$;

$\mathrm{a}_{3}=0,065649$;

$a_{4}=(-0,0059684)$;

$\mathrm{a}_{5}=(-0,0005967)$;

$\mathrm{UR}_{\mathrm{MIN}}=$ umidade relativa mínima do ar (\%);

$\mathrm{n}=$ número de horas reais de insolação $(\mathrm{h})$;

$\mathrm{N}=$ fotoperíodo $(\mathrm{h}) ; \mathrm{e}$

$\mathrm{Ud}=$ velocidade do vento do período diurno a $2 \mathrm{~m}$ de altura $\left(\mathrm{m} \mathrm{s}^{-1}\right)$.

\section{Método da Radiação (FAO 24)}

A equação da radiação (FAO 24)desenvolvida por Doorenbos e Pruitt (1977) é expressa da seguinte maneira.

$$
E T=r W R_{s}
$$

em que

$r=$ é um fator de ajuste que varia com a umidade relativa média e a velocidade do vento (FREVERT et al., 1983);

$\mathrm{W}=$ é um fator de ponderação, que inclui os 
efeitos da temperatura e da altitude na relação entre a radiação da superfície do solo e da evapotranspiração de referência (DOORENBOS E PRUITT, 1977); e

Rs $=$ é a radiação solar $\left(\mathrm{mm} \cdot \mathrm{d}^{-1}\right)$.

\section{Método de Priestley-Taylor}

Priestley e Taylor (1972) mostraram que, sob certas condições, o conhecimento do saldo da radiação e da aridez do solo é suficiente para determinar os fluxos de calor sensível e de vapor na superfície terrestre. O método pode ser considerado um balanço de energia com base apenas no termo radiativo (diabático) corrigido por um fator de ajuste, cuja equação é a seguinte.

$$
E T=1,26 \frac{\Delta}{\Delta+\gamma} \cdot \frac{(R n-G)}{\lambda}
$$

em que

$\mathrm{ET}=$ evapotranspiração estimada pelo método de Priestley-Taylor $\left(\mathrm{mm} \mathrm{d}^{-1}\right)$;

$\mathrm{Rn}=$ saldo de radiação da superfície evaporante, $\left(\mathrm{MJ} \mathrm{m}^{-2} \mathrm{~d}^{-1}\right)$;

$\mathrm{G}=$ densidade de fluxo de calor sensível no solo, $\left(\mathrm{MJ} \mathrm{m}^{-2} \mathrm{~d}^{-1}\right)$;

$\Delta=$ declividade da curva de pressão de vapor de saturação $\left(\mathrm{kPa}^{\circ} \mathrm{C}^{-1}\right)$;

$\gamma=$ coeficiente psicrométrico $\left(\mathrm{kPa}^{\circ} \mathrm{C}^{-1}\right)$; e

$\lambda=$ calor latente de vaporização $\left(\mathrm{MJ} \mathrm{kg}^{-1}\right)$.

\section{Método de Hargreaves-Samani}

O método proposto por Hargreaves-Samani (1985) para estimativa da evapotranspiração de referência é uma alternativa para situações em que não há medições da superfície dos dados da radiação solar, da umidade relativa do ar e da velocidade do vento:

$$
E T=0,0023\left(T_{\max }-T_{\min }\right)^{0,5}\left(T_{\text {med }}+17,8\right) R a
$$

em que

$\mathrm{T}_{\max }=$ é a temperatura máxima do dia $\left({ }^{\circ} \mathrm{C}\right)$;

$\mathrm{T}_{\min }=$ é a temperatura mínima do dia $\left({ }^{\circ} \mathrm{C}\right)$;
$\mathrm{T}_{\text {med }}=$ é a temperatura média do dia $\left({ }^{\circ} \mathrm{C}\right)$; e

$\mathrm{Ra}=$ é a radiação extraterrestre, $\left(\mathrm{mm} \mathrm{d}^{-1}\right)$.

\section{Método de Penman-Monteith}

Para o cálculo da evapotranspiração, utilizouse o método de Penman-Monteith (FAO), parametrizada por Allen et al. (2006), para intervalo de tempo de 24 horas.

$$
E T_{0}=\frac{0,408 \Delta\left(R_{\mathrm{n}}-G\right)+\gamma \frac{900}{T+273} u_{2}(e s-e a)}{\Delta+\gamma\left(1+0,34 u_{2}\right)} \text { (9) }
$$

em que

ETo $=$ evapotranspiração de referência $\left(\mathrm{mm}^{\mathrm{d}} \mathrm{d}^{-1}\right)$;

$\mathrm{Rn}=$ saldo de radiação à superfície $\left(\mathrm{MJ} \mathrm{m}^{-2} \mathrm{~d}^{-1}\right)$;

$\mathrm{G}=$ densidade do fluxo de calor no solo $\left(\mathrm{MJ} \mathrm{m}^{-2} \mathrm{~d}^{-1}\right)$;

$\mathrm{T}=$ temperatura do ar a $2 \mathrm{~m}$ de altura $\left({ }^{\circ} \mathrm{C}\right)$;

$\mathrm{u}_{2}=$ velocidade do vento a $2 \mathrm{~m}$ de altura $\left(\mathrm{m} \mathrm{s}^{-1}\right)$;

es = pressão de vapor de saturação $(\mathrm{kPa})$;

ea $=$ pressão parcial de vapor $(\mathrm{kPa})$;

$\Delta=$ declividade da curva de pressão de vapor de saturação $\left(\mathrm{kPa}^{\circ} \mathrm{C}^{-1}\right)$; e

$\gamma=$ coeficiente psicrométrico $\left(\mathrm{kPa}^{\circ} \mathrm{C}^{-1}\right)$.

\section{Avaliação dos métodos}

A análise de desempenho dos métodos foi feita pela comparação dos valores de ET obtidos pelo método padrão de Penman-Monteith (FAO56) com aqueles obtidos pelos outros quatro métodos (Blaney Criddle - FAO 24, Radiação - FAO 24, Priestley-Taylor e Hargreaves-Samani).

Os valores de ETo obtidos pelo método de Penman-Monteith (FAO56) e os computados pelos outros métodos foram comparados por meio: (1) da equação de regressão linear $(\mathrm{Y}=\mathrm{a}+\mathrm{bX})$, em que $\mathrm{Y}$ é a evapotranspiração obtida do método avaliado e X é a evapotranspiração obtida pelo método padrão; (2) do coeficiente de determinação $\left(\mathrm{R}^{2}\right)$; (3) da raiz do erro quadrático médio (REQM); (4) do erro absoluto médio (EAM); (5) do índice de concordância de Willmott; e (6) do índice de desempenho (c). No caso de uma correlação perfeita, sem viés, $a=0$ e $b$ $=1, \mathrm{R}^{2}=1, \mathrm{EAM}=0$ e $\mathrm{REQM}=0$.

Os erros dos modelos foram calculados pela 
raiz do erro quadrático médio (REQM) e pelo erro absoluto médio (EAM) dados pelas equações:

$$
\begin{aligned}
& \text { REQM }=\sqrt{\left(\mathrm{N}^{-1} \sum_{\mathrm{i}=1}^{\mathrm{N}}\left(\mathrm{P}_{\mathrm{i}}-\mathrm{O}_{\mathrm{i}}\right)^{2}\right)} \\
& \mathrm{EAM}=\mathrm{N}^{-1} \sum_{\mathrm{i}=1}^{\mathrm{N}}\left|\mathrm{P}_{\mathrm{i}}-\mathrm{O}_{\mathrm{i}}\right|
\end{aligned}
$$

em que

$\mathrm{N}$ = número de observações; Pi são valores estimados da ET $\left(\mathrm{mm} \mathrm{d}^{-1}\right)$; e

Oi $=$ valores de ETo calculados pelo método PM FAO $56\left(\mathrm{~mm} \mathrm{~d}^{-1}\right)$.

O estudo comparativo entre os métodos estudados e o método padrão foi realizado pelo índice de concordância (d) proposto por Willmott 1982.

$$
d=1-\left[\frac{\sum_{i=1}^{N}\left(P_{i}-O_{i}\right)^{2}}{\sum_{i=1}^{N}\left(\left|P_{i}-O\right|-\left|O_{i}-O\right|\right)^{2}}\right]
$$

em que

$\mathrm{O}=$ média dos valores de ETo obtida pelo método padrão, $\left(\mathrm{mm}^{\mathrm{d}} \mathrm{d}^{-1}\right)$.

Esta medida condensa todas as diferenças entre as estimativas do modelo e as observações em uma estatística quantitativa. Visto de outra perspectiva, o índice de concordância é uma medida de quão bem o modelo estima o afastamento dos dados da média observada. O índice de concordância tem um intervalo variando entre 0 e 1 , sendo que valores próximos a 1 mostram uma concordância perfeita.

Analogamente, para a análise da confiabilidade de um modelo, considerou-se o índice de confiança c proposto por Camargo e Sentelhas (1997):

$\mathrm{c}=\mathrm{r} \times \mathrm{d}$

em que

$\mathrm{r}=$ coeficiente de correlação de Pearson; e

$\mathrm{d}=$ índice de concordância de Willmott.

Um índice $\mathrm{c}=0$ indica confiança nula e $\mathrm{c}=$ 1 significa confiança perfeita. $\mathrm{O}$ critério adotado para interpretar o desempenho dos métodos pelo índice "c" para os valores médios diários de evapotranspiração está no Quadro 1.

\section{RESULTADOS E DISCUSSÃO}

Na Figura 1, dividida em A, B e C, estão mostradas as variações médias mensais da evapotranspiração para os métodos estudados nas localidades de Espinosa, Montes Claros e Salinas, respectivamente.

Em relação ao método de Penman-Monteith (FAO 56), as estimativas feitas pelos métodos da Radiação e de Hargreaves-Samani tenderam a superestimar em todas as localidades. Porém, para a cidade de Salinas, nos meses de abril a setembro, o método da Radiação apresentou valores próximos do método de Penman-Monteith.

Nas três localidades estudadas, o método de

Quadro 1. Critério de interpretação do desempenho dos métodos de estimativa da ETo pelo índice "c" proposto por Camargo e Sentelhas (1997)

\begin{tabular}{cc}
\hline Valor de "c" & Desempenho \\
\hline$>0,85$ & Ótimo \\
0,76 a 0,85 & Muito Bom \\
0,66 a 0,75 & Bom \\
0,61 a 0,65 & Mediano \\
0,51 a 0,60 & Sofrível \\
0,41 a 0,50 & Ruim \\
$\leq 0,40$ & Péssimo \\
\hline
\end{tabular}



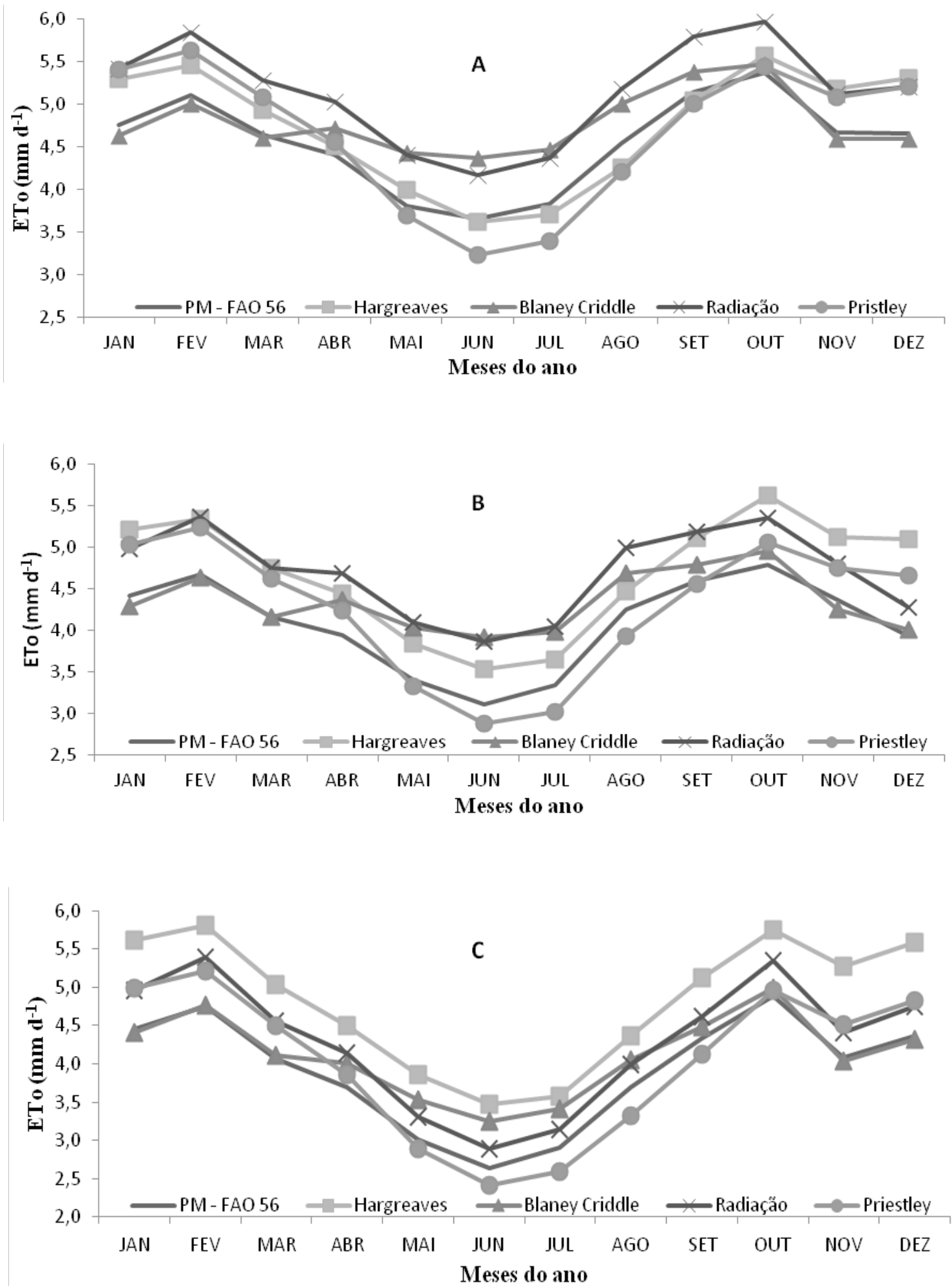

Figura 1. Valores médios mensais da evapotranspiração para os métodos estudados para a localidade de Espinosa (A), Montes Claros (B) e Salinas (C). 
Blaney Criddle apresenta valores próximos ao de Penman-Monteith (FAO 56) nos meses de outubro a março, e nos meses de abril a setembro ele tende a superestimar os valores do método padrão. Além disso, na Figura 1, pode-se observar que, em relação ao método de Penman-Monteith (FAO 56), a ETo obtida por meio do método de Priestley-Taylor apresentou valores superestimados nos meses de outubro a março e subestimados nos meses de abril a março.

\section{Avaliação dos métodos considerando o ano completo}

Os dados diários de ETo calculados pelo método de Penman-Monteith (FAO 56) foram comparados com os valores calculados pelos outros métodos estudados. Esta comparação foi realizada a partir das observações diárias realizadas num período de 10 anos.

A simples adoção do coeficiente de determinação $\left(\mathrm{R}^{2}\right)$ como único critério de definição da qualidade de métodos não é adequado, uma vez que esse método não estabelece o tipo e a magnitude das diferenças entre um valor padrão e um valor previsto por modelos de estimativa (BARROS et al., 2009). Assim, a análise dos índices de concordância de Wilmont (d), erro absoluto médio (EAM) e a raiz do erro quadrático médio (REQM) auxiliam a interpretação dos resultados.

Os resultados da comparação através da análise de regressão simples entre os valores calculados por diversos métodos e o método padrão, mostrando o erro associado a cada método através dos índices estatísticos, estão apresentados no Quadro 2. Os quatro métodos de cálculo foram considerados variáveis dependentes e os valores do método padrão, a variável independente.

Os valores para os parâmetros da equação de regressão $(\mathrm{a}$ e $\mathrm{b}$ ) foram altamente significativos ( $\mathrm{P}$ $<0,01)$ em todos os casos.

Para a cidade de Espinosa, o método de Hargreaves-Samani teve o pior desempenho entre os métodos avaliados, superestimando em relação ao método de Penman-Monteith (FAO 56), com um erro absoluto médio de $0,62 \mathrm{~mm} \mathrm{~d}^{-1}$. Além de elevados erros, este método apresentou o pior coeficiente de determinação, sendo classificado como bom pelo índice de confiança c proposto por Camargo e Sentelhas (1997). Entre os métodos avaliados, o de Blaney Criddle apresentou o melhor desempenho e os menores erros, podendo ser classificado como ótimo para o período analisado. Já o método de Priestley-Taylor teve bom desempenho, sendo que os coeficientes da equação de regressão apresentaram valores próximos do ideal. Este método também apresentou valor médio de ETo mais próximo do método de PenmanMonteith (FAO 56), com erro absoluto médio de $0,44 \mathrm{~mm} \mathrm{~d}^{-1}$.

Quanto ao método da Radiação, observou-se um resultado atípico, com os maiores erros e a maior superestimativa (13\%). Porém, o método apresenta elevados valores do coeficiente de determinação $(0,934)$ e índice de concordância $(0,92)$. O método apresenta uma boa concordância com o método de Penman-Monteith (FAO 56), podendo ser considerado como ótimo, mesmo apresentando erros maiores que o método de Hargreaves-Samani.

Para a cidade de Montes Claros, o método de Priestley-Taylor teve o melhor desempenho entre os métodos avaliados, com os menores erros e a menor superestimativa, seguido do método de Blaney Criddle e da Radiação. O método de HargreavesSamani teve o pior desempenho levando em consideração o índice de concordância e o índice de confiança c, podendo ser classificado como bom. Novamente, o método da Radiação apresentou os maiores erros e a maior superestimativa.

Os resultados obtidos para a cidade de Salinas mostram um comportamento próximo ao verificado para as outras cidades, sendo que o método de Blaney Criddle é o melhor, seguido dos métodos de Priestley-Taylor, Radiação e Hargreaves.

Tagliaferre et al. (2010) compararam diferentes métodos de estimativa de evapotranspiração de referência para a região de Eunápolis-BA, tendo verificado que os métodos de Blaney-Criddle, Radiação e Priestley Taylor apresentam melhor desempenho para estimativa de ETo em todos os períodos de tempo estudado e também que o método de Hargreaves-Samani foi o pior método usado na estimativa da evapotranspiração de referência.

Como o método de Hargreaves-Samani leva em consideração apenas as temperaturas máximas 
e mínimas, a influência das outras variáveis climáticas na evapotranspiração não pode ser observada por este método.

Resultados parecidos com os obtidos neste trabalho, para o método de Hargreaves-Samani, foram encontrados por Reis et al. (2007) quando avaliaram o desempenho de métodos empíricos para estimativa da evapotranspiração de referência para três localidades do Estado do Espírito Santo.

\section{Avaliação dos métodos para os períodos de alta e baixa demanda evaporativa}

A relação entre os valores do método padrão e os diferentes métodos para estimar a ETo diária também foi avaliada em dois períodos diferentes do ano: um para o período de baixa demanda evaporativa compreendido entre os meses de abril a setembro e outro de alta demanda compreendido entre outubro a março. Estes dois períodos do ano foram diferenciados com o objetivo de descobrir o método mais adequado para cada um dos períodos estudados.

No Quadro 3 mostram-se os resultados da comparação entre os métodos estudados e o método padrão para o período de outubro a março.

Quadro 2. Avaliação dos métodos de cálculo da evapotranspiração de referência considerando o ano completo

Espinosa

\begin{tabular}{|c|c|c|c|c|c|c|c|c|c|}
\hline \multirow[t]{2}{*}{ Modelo } & \multicolumn{3}{|c|}{$\begin{array}{c}\text { ET(est) }=a+b \text { ETo } \\
(\mathrm{PM})\end{array}$} & \multicolumn{6}{|c|}{ Parâmetros } \\
\hline & $\mathrm{a}$ & $\mathrm{b}$ & $\mathrm{R}^{2}$ & $\mathrm{r}$ & REQM & EAM & $\mathrm{d}$ & $\mathrm{c}$ & Média \\
\hline Penman-Monteith & & & & & & & & & 4,54 \\
\hline Hargreaves-Samani & 1,725 & 0,662 & 0,601 & 0,775 & 0,76 & 0,62 & 0,87 & 0,67 & 4,73 \\
\hline Blaney Criddle & 0,297 & 0,983 & 0,896 & 0,947 & 0,45 & 0,34 & 0,96 & 0,91 & 4,77 \\
\hline Priestley-Taylor & 0,063 & 1,009 & 0,831 & 0,912 & 0,54 & 0,44 & 0,95 & 0,87 & 4,65 \\
\hline Radiação & 0,606 & 1,265 & 0,934 & 0,966 & 0,78 & 0,68 & 0,92 & 0,89 & 5,14 \\
\hline \multicolumn{10}{|c|}{ Montes Claros } \\
\hline \multirow{2}{*}{ Modelo } & \multicolumn{3}{|c|}{$\begin{array}{c}\mathrm{ET}(\text { est })=\mathrm{a}+\mathrm{b} \text { ETo } \\
(\mathrm{PM})\end{array}$} & \multicolumn{6}{|c|}{ Parâmetros } \\
\hline & $\mathrm{a}$ & $\mathrm{b}$ & $\mathrm{R}^{2}$ & $\mathrm{r}$ & REQM & EAM & $\mathrm{d}$ & $\mathrm{c}$ & Média \\
\hline Penman-Monteith & & & & & & & & & 4,10 \\
\hline Hargreaves-Samani & 1,395 & 0,799 & 0,744 & 0,863 & 0,80 & 0,64 & 0,86 & 0,75 & 4,67 \\
\hline Blaney Criddle & 0,326 & 0,977 & 0,872 & 0,934 & 0,48 & 0,37 & 0,96 & 0,89 & 4,33 \\
\hline Priestley-Taylor & 0,197 & 1,088 & 0,902 & 0,950 & 0,44 & 0,35 & 0,97 & 0,92 & 4,26 \\
\hline Radiação & 0,662 & 1,316 & 0,926 & 0,962 & 0,83 & 0,72 & 0,90 & 0,87 & 4,73 \\
\hline \multicolumn{10}{|c|}{ Salinas } \\
\hline \multirow[t]{2}{*}{ Modelo } & \multicolumn{3}{|c|}{$\begin{array}{c}\mathrm{ET}(\text { est })=\mathrm{a}+\mathrm{b} \text { ETo } \\
(\mathrm{PM})\end{array}$} & \multicolumn{6}{|c|}{ Parâmetros } \\
\hline & $\mathrm{a}$ & $\mathrm{b}$ & $\mathrm{R}^{2}$ & $\mathrm{r}$ & REQM & EAM & $\mathrm{d}$ & $\mathrm{c}$ & Média \\
\hline Penman-Monteith & & & & & & & & & 3,90 \\
\hline Hargreaves-Samani & 1,466 & 0,860 & 0,799 & 0,894 & 1,07 & 0,95 & 0,83 & 0,74 & 4,82 \\
\hline Blaney Criddle & 0,155 & 1,013 & 0,918 & 0,958 & 0,42 & 0,32 & 0,97 & 0,93 & 4,10 \\
\hline Priestley-Taylor & 0,025 & 1,034 & 0,875 & 0,935 & 0,50 & 0,39 & 0,96 & 0,90 & 4,01 \\
\hline Radiação & 0,750 & 1,291 & 0,936 & 0,967 & 0,67 & 0,56 & 0,95 & 0,92 & 4,29 \\
\hline
\end{tabular}

a - coeficiente linear da equação de regressão, $b$ - coeficiente angular da equação de regressão, $\mathrm{R}^{2}-$ coeficiente de determinação, $r$ - coeficiente de correlação de Pearson, REQM - raiz do erro quadrático médio, EAM - erro absoluto médio, $\mathrm{d}$ - índice de concordância de Willmott, c - índice de confiança 
Quadro 3. Avaliação dos métodos de cálculo da evapotranspiração de referência para o período de alta demanda evaporativa

\begin{tabular}{|c|c|c|c|c|c|c|c|c|c|}
\hline \multicolumn{10}{|c|}{ Espinosa } \\
\hline \multirow[t]{2}{*}{ Modelo } & \multicolumn{3}{|c|}{$\begin{array}{c}\mathrm{ET}(\text { est })=\mathrm{a}+\mathrm{b} \text { ETo } \\
(\mathrm{PM})\end{array}$} & \multicolumn{6}{|c|}{ Parâmetros } \\
\hline & $\mathrm{a}$ & $\mathrm{b}$ & $\mathrm{R}^{2}$ & $\mathrm{r}$ & REQM & EAM & $\mathrm{d}$ & $\mathrm{c}$ & Média \\
\hline Penman-Monteith & & & & & & & & & 4,86 \\
\hline Hargreaves-Samani & 2,547 & 0,562 & 0,672 & 0,820 & 0,87 & 0,71 & 0,84 & 0,69 & 5,28 \\
\hline Blaney Criddle & 0,556 & 1,104 & 0,987 & 0,993 & 0,21 & 0,17 & 0,99 & 0,99 & 4,81 \\
\hline Priestley-Taylor & 0,622 & 0,962 & 0,918 & 0,958 & 0,58 & 0,50 & 0,95 & 0,91 & 5,30 \\
\hline Radiação & $-1,110$ & 1,353 & 0,966 & 0,983 & 0,82 & 0,70 & 0,93 & 0,91 & 5,47 \\
\hline
\end{tabular}

Montes Claros

$\mathrm{ET}(\mathrm{est})=\mathrm{a}+\mathrm{b}$ ETo

Modelo

$(\mathrm{PM})$

Parâmetros

\begin{tabular}{|c|c|c|c|c|c|c|c|c|c|}
\hline \multirow[t]{2}{*}{ Modelo } & \multicolumn{3}{|c|}{ (PM) } & \multirow[b]{2}{*}{$\mathrm{r}$} & \multirow[b]{2}{*}{ REQM } & \multirow[b]{2}{*}{ EAM } & \multirow[b]{2}{*}{$\mathrm{d}$} & \multirow[b]{2}{*}{$\mathrm{c}$} & \multirow[b]{2}{*}{ Média } \\
\hline & $\mathrm{a}$ & $\mathrm{b}$ & $\mathrm{R}^{2}$ & & & & & & \\
\hline Penman-Monteith & & & & & & & & & 4,45 \\
\hline Hargreaves-Samani & 2,148 & 0,683 & 0,753 & 0,868 & 0,97 & 0,80 & 0,83 & 0,72 & 5,18 \\
\hline Blaney Criddle & 0,603 & 1,121 & 0,990 & 0,995 & 0,21 & 0,18 & 0,99 & 0,99 & 4,37 \\
\hline Priestley-Taylor & 0,322 & 1,025 & 0,947 & 0,973 & 0,53 & 0,46 & 0,96 & 0,93 & 4,88 \\
\hline Radiação & 1,459 & 1,451 & 0,979 & 0,989 & 0,82 & 0,67 & 0,93 & 0,92 & 4,99 \\
\hline
\end{tabular}

Salinas

\begin{tabular}{cccccccccccc}
\hline \multirow{2}{*}{ Modelo } & \multicolumn{3}{c}{$\begin{array}{c}\text { ET (est) }=\mathrm{a}+\mathrm{b} \text { ETo } \\
(\mathrm{PM})\end{array}$} & \multicolumn{4}{c}{ Parâmetros } \\
\cline { 2 - 11 } & $\mathrm{a}$ & $\mathrm{b}$ & $\mathrm{R}^{2}$ & $\mathrm{r}$ & REQM & EAM & $\mathrm{d}$ & $\mathrm{c}$ & Média \\
\hline Penman-Monteith & & & & & & & & & 4,43 \\
Hargreaves-Samani & 2,217 & 0,741 & 0,775 & 0,880 & 1,21 & 1,08 & 0,77 & 0,68 & 5,50 \\
Blaney Criddle & 0,589 & 1,133 & 0,986 & 0,993 & 0,23 & 0,19 & 0,99 & 0,99 & 4,43 \\
Priestley-Taylor & 0,512 & 0,974 & 0,923 & 0,961 & 0,52 & 0,46 & 0,95 & 0,92 & 4,83 \\
Radiação & 1,250 & 1,386 & 0,967 & 0,983 & 0,73 & 0,61 & 0,94 & 0,92 & 4,90 \\
\hline
\end{tabular}

$\mathrm{a}$ - coeficiente linear da equação de regressão, $\mathrm{b}$ - coeficiente angular da equação de regressão, $\mathrm{R}^{2}$ - coeficiente de determinação, $r$ - coeficiente de correlação de Pearson, REQM - raiz do erro quadrático médio, EAM - erro absoluto médio, $\mathrm{d}$ - índice de concordância de Willmott, $\mathrm{c}$ - índice de confiança

Os métodos de Hargreaves-Samani e da Radiação superestimaram o método padrão para todas as localidades, tendo novamente apresentado os maiores valores de EAM e REQM e mostrado os menores índices de concordância de Willmott e as maiores superestimativas em relação à média. O método de Hargreaves-Samani pode ser classificado como bom, e o método da Radiação como ótimo baseado no índice de confiança c.

O método de Blaney Criddle é o melhor método para estimativa da ET para o período de alta demanda evaporativa. A inclinação da reta da linha de regressão é próxima de 1 , indicando o desempenho correto do método de Blaney Criddle para este intervalo de valores. Este método apresentou os maiores valores do coeficiente de determinação e do índice de concordância de Willmott e os menores valores de EAM e REQM, mostrando uma boa concordância com os dados do método padrão de Penman-Monteith (FAO 56), o que pode ser observado na Figura 1, sendo que neste período a média é próxima da média do método padrão. 
Para as localidades de Espinosa e Montes Claros, a média obtida pelo método de Blaney Criddle para o período de alta demanda foi menor que a ETo obtida pelo método de Penman-Monteith (FAO 56). O método de Priestley-Taylor teve um desempenho pouco melhor que o método da Radiação, apresentando menores erros e um maior índice de concordância de Willmott, podendo ser classificado com ótimo, baseado no índice c.

No Quadro 4 estão mostrados os resultados da comparação entre os métodos estudados e o método padrão para o período de baixa demanda evaporativa (abril a setembro).
Os métodos de Blaney Criddle e de PriestleyTaylor apresentaram os melhores desempenhos na estimativa da ET para o período de abril a setembro (baixa demanda evaporativa). Porém estes métodos têm seu desempenho aquém quando comparados com o período de alta demanda, podendo-se observar que os métodos de Blaney Criddle e de Priestley-Taylor apresentam um melhor desempenho na estimativa da ET nos meses de outubro a março.

O método de Blaney Criddle apresenta uma superestimativa em relação ao métode de PenmanMonteith em todas as localidades, podendo ser

Quadro 4. Avaliação dos métodos de cálculo da evapotranspiração de referência para o período de baixa demanda evaporativa

\begin{tabular}{|c|c|c|c|c|c|c|c|c|c|}
\hline \multicolumn{10}{|c|}{ Espinosa } \\
\hline \multirow[t]{2}{*}{ Modelo } & \multicolumn{3}{|c|}{$\begin{array}{c}\text { ET(est) }=a+b \text { ETo } \\
(\mathrm{PM})\end{array}$} & \multicolumn{6}{|c|}{ Parâmetros } \\
\hline & $\mathrm{A}$ & $\mathrm{b}$ & $\mathrm{R}^{2}$ & $\mathrm{r}$ & REQM & EAM & $\mathrm{d}$ & $\mathrm{c}$ & Média \\
\hline Penman-Monteith & & & & & & & & & 4,22 \\
\hline Hargreaves-Samani & 1,649 & 0,599 & 0,518 & 0,720 & 0,66 & 0,54 & 0,84 & 0,60 & 4,18 \\
\hline Blaney Criddle & 0,717 & 0,947 & 0,874 & 0,935 & 0,58 & 0,49 & 0,90 & 0,84 & 4,72 \\
\hline Priestley-Taylor & 0,397 & 0,853 & 0,767 & 0,876 & 0,50 & 0,39 & 0,92 & 0,81 & 4,00 \\
\hline Radiação & 0,036 & 1,148 & 0,871 & 0,933 & 0,73 & 0,65 & 0,88 & 0,82 & 4,81 \\
\hline \multicolumn{10}{|c|}{ Montes Claros } \\
\hline \multirow[t]{2}{*}{ Modelo } & ET(e & $\begin{array}{l}=a+ \\
(P M)\end{array}$ & ETo & \multicolumn{6}{|c|}{ Parâmetros } \\
\hline & $\mathrm{A}$ & $\mathrm{b}$ & $\mathrm{R}^{2}$ & $\mathrm{r}$ & REQM & EAM & $\mathrm{d}$ & $\mathrm{c}$ & Média \\
\hline Penman-Monteith & & & & & & & & & 3,74 \\
\hline Hargreaves-Samani & 1,103 & 0,812 & 0,726 & 0,852 & 0,59 & 0,47 & 0,87 & 0,74 & 4,14 \\
\hline Blaney Criddle & 0,692 & 0,960 & 0,837 & 0,915 & 0,64 & 0,56 & 0,87 & 0,79 & 4,28 \\
\hline Priestley-Taylor & 0,009 & 0,972 & 0,876 & 0,936 & 0,32 & 0,25 & 0,96 & 0,90 & 3,62 \\
\hline Radiação & 0,079 & 1,214 & 0,862 & 0,928 & 0,84 & 0,77 & 0,83 & 0,77 & 4,46 \\
\hline
\end{tabular}

Salinas

\begin{tabular}{cccccccccccc}
\hline & \multicolumn{3}{c}{$\begin{array}{c}\text { ET(est)= a }+\mathrm{b} \text { ETo } \\
\text { Modelo }\end{array}$} & \multicolumn{3}{c}{ (PM) } & \multicolumn{4}{c}{ Parâmetros } \\
\cline { 2 - 11 } & $\mathrm{a}$ & $\mathrm{b}$ & $\mathrm{R}^{2}$ & $\mathrm{r}$ & REQM & EAM & $\mathrm{d}$ & $\mathrm{c}$ & Média \\
\hline Penman-Monteith & & & & & & & & & 3,37 \\
Hargreaves-Samani & 1,456 & 0,797 & 0,755 & 0,869 & 0,92 & 0,81 & 0,81 & 0,70 & 4,14 \\
Blaney Criddle & 0,200 & 1,062 & 0,892 & 0,944 & 0,55 & 0,45 & 0,93 & 0,88 & 3,77 \\
Priestley-Taylor & 0,372 & 0,836 & 0,814 & 0,902 & 0,46 & 0,33 & 0,94 & 0,85 & 3,18 \\
Radiação & 0,476 & 1,233 & 0,874 & 0,935 & 0,61 & 0,50 & 0,93 & 0,87 & 3,67 \\
\hline
\end{tabular}

$\mathrm{a}$ - coeficiente linear da equação de regressão, $\mathrm{b}$ - coeficiente angular da equação de regressão, $\mathrm{R}^{2}$ - coeficiente de determinação, $\mathrm{r}$ - coeficiente de correlação de Pearson, REQM - raiz do erro quadrático médio, EAM - erro absoluto médio, $\mathrm{d}$ - índice de concordância de Willmott, $\mathrm{c}$ - índice de confiança 
classificado como muito bom para Espinosa e Montes Claros e ótimo para Salinas. O método de Priestley-Taylor apresenta uma subestimativa do método de Penman-Monteith e os menores erros, podendo ser classificado como ótimo para Montes Claros e Salinas e muito bom para Espinosa. Para este método, a raiz do erro quadrático médio variou de 0,32 a $0,50 \mathrm{~mm} \mathrm{dia}^{-1}$ e o índice de concordância entre 0,92 a 0,94.

O método de Hargreaves-Samani apresentou uma melhora no desempenho quando comparado com o período de alta demanda, podendo ser classificado como bom para Montes Claros e Salinas e sofrível para a cidade de Espinosa. O erro absoluto médio do método de HargreavesSamani variou de 0,47 a $0,81 \mathrm{~mm} \mathrm{dia}{ }^{-1}$ e apresentou uma pequena subestimativa para a cidade de Espinosa.

Como o método de Hargreaves-Samani foi obtido para a condição semiárida do Nordeste brasileiro, seu desempenho apresenta uma melhora na estimativa da evapotranspiração nos meses mais seco (outubro a março).

O método da Radiação apresentou uma piora no desempenho em relação ao período de alta demanda. As médias da ETo para as três localidades foram superiores àquelas do método padrão de Penman-Monteith. Nas cidades de Espinosa e Montes Claros, o método da radiação apresentou os maiores erros. O método pode ser classificado como muito bom em Espinosa e Montes Claros e ótimo para Salinas. LópezUrrea et al. (2006) avaliaram diferentes métodos de estimativa da evapotranspiração para uma região semiárida da Espanha, tendo observado que o método da Radiação superestimou a ET obtida pelo método de Penman-Monteith (FAO 56).

$\mathrm{Na}$ comparação com o período de alta demanda evaporativa, pode-se observar uma diminuição no coeficiente de determinação em todos os métodos avaliados no período de baixa demanda evaporativa, devido a um aumento da dispersão dos valores em relação à ETo do método padrão de Penman-Monteith (FAO 56).

O bom desempenho obtido pelo método de Blaney Criddle pode ser explicado pelo fato de o método ter sido desenvolvido para condições semiáridas dos Estados Unidos, ajustando-se bem às condições do clima da região em estudo.

Apesar de o método de Hargreaves-Samani ter mostrado um desempenho inferior ao método de Penman-Monteith (FAO 56), ele constitui uma boa solução para estimativas de ET na ausência de dados de radiação, velocidade do vento e umidade relativa do ar.

A avaliação comparativa de modelos para locais diferentes mostra desvios no desempenho de cada método, que podem ser atribuídos a diferenças na formulação das variáveis de cada modelo.

\section{CONCLUSÕES}

- Para as localidades estudadas, os métodos de Blaney Criddle e Priestley-Taylor foram os mais adequados na estimativa da evapotranspiração quando comparados com o método de Penman-Monteith (FAO 56), podendo ser classificados como ótimos para o período anual;

- O método de Hargreaves-Samani apresentou o pior desempenho entre os métodos avaliados para o período de outubro a março (alta demanda);e

- O método da Radiação apresentou o pior desempenho entre os métodos avaliados para o período de baixa demanda evaporativa (abril a setembro), tendo sido propenso a superestimar o método de Penman-Monteith (FAO 56) em todos os períodos estudados.

\section{AGRADECIMENTO}

Agradecemos ao $\mathrm{CNPq}$ - Conselho Nacional de Desenvolvimento Científico e Tecnológico, pelo apoio ao desenvolvimento deste trabalho. Agradecemos ainda ao INMET - Instituto Nacional de Meteorologia, pela disponibilização dos dados utilizados.

\section{REFERÊNCIAS BIBLIOGRÁFICAS}

AHMADI, S.H.; FOOLADMAND, H.R. Spatially distributed monthly reference evapotranspiration 
derived from the calibration of Thornthwaite equation: a case study, South of Iran. Irrigation Science, v.26(4), p.303-312. 2008.

ALLEN, R.G.; PEREIRA, L.S.; RAES, D.; SMITH, J. Evapotranspiration del cultivo: guias para la determinación de los requerimientos de agua de los cultivos. Roma: FAO, 2006. 298 p. (Estudio Riego e Drenaje Paper, 56).

BARROS, V. R.; SOUZA, A.P.; FONSECA, D.C.; SILVA, L.B.D. Avaliação da evapotranspiração de referência na Região de Seropédica, Rio de Janeiro, utilizando lisímetro de pesagem e modelos matemáticos. Revista Brasileira de Ciências Agrárias, v.4, n.2, p.198-203, 2009.

BLANEY, H.F.; CRIDDLE, W.D. Determining water requirements in irrigated areas from climatological and irrigation data. In: USDA Soil Conserv. Serv., SCS-TP-96, 44p. 1950.

CAMARGO, A.P.; SENTELHAS, P.C. Avaliação do desempenho de diferentes métodos de estimativa da evapotranspiração potencial no Estado de São Paulo, Brasil. Rev. Bras. Agrometeorol., Santa Maria, v.5, n.1, p.89-97, 1997.

DOORENBOS, J.; PRUITT, W.O. Crop Water Requirements. FAO, Rome, FAO Irrigation and Drainage, Paper no. 24. 1977.

FOOLADMAND, H.R.; AHMADI, S.H. Monthly spatial calibration of Blaney-Criddle equation for calculating monthly ETo in south of Iran. Irrigation and Drainage, v.58(2), p.234-245, 2009.

FREVERT, D.K.; HILL, R.W.; BRAATEN, B.C. Estimation of FAO evapotranspiration coefficients. J. Irrig. Drain. Eng. ASCE 109 (IR2), p.265-270. 1983.

GAVILÁN, P.; LORITE, I.J.; TORNERO, S.; BERENGENA, J. Regional calibration of Hargreaves equation for estimating reference ET in a semiarid environment. Agriculture Water
Management., v.81, p.257-281, 2006.

HARGREAVES, G.H.; SAMANI, Z.A., Reference crop evapotranspiration from temperature. Appl. Eng. Agric, v.1, n.2, p.96-99, 1985.

LIANG, L.; LIJUAN, L.; ZHANG, L.; LI, J. Sensitivity of Penman-Monteith reference crop evapotranspiration in Tao'er River Basin of northeastern China. Chinese Geographical Science, v. 18, n.4, p.340-347, 2008.

LÓPEZ-URREA, R.; MARTIN DE SANTA OLALLA, F.; FABEIRO, C.; MORATALLA, A. Testing Evapotranspiration Equations Using Lysimeter Observations in a Semiarid Climate. J. Agric. Water Manag.,v. 85. pp. 15-26. 2006.

MAKKINK, G.F. Testing the Penman formula by means of lysimeters, J. Inst. Water Eng., v.11, p.277-288, 1957.

MAEDA, E.E.; WIBERG, D.A.; PELLIKKA, P.K.E. Estimating reference evapotranspiration using remote sensing and empirical models in a region with limited ground data availability in Kenya. Applied Geography. doi:10.1016/j. apgeog.2010.05.011

OLIVEIRA, L.M.M. de.; MONTENEGRO, S. M.G.L.; AZEVEDO, J.R.G. de; SANTOS, F. $X$. Evapotranspiração de referência na bacia experimental do riacho Gameleira, PE, utilizando lisímetro e métodos indiretos. Revista Brasileira de Ciências Agrárias, v.3, n.1, p.58-67, 2008.

ORTEGA-FARIAS, S.; IRMAK, S.; CUENCA, R.H. Special issue on evapotranspiration measurement and modeling, Irrigation Science v.28, n.1, p.1-3, 2009.

PRIESTLEY, C.H.B., TAYLOR R.J. On the assessment of surface heat flux and evaporation using large scale parameters, Mon. Weather Rev. v.100, n.2, p.81-92, 1972.

REIS, E. F. dos.; BRAGANÇA, R.; GARCIA, G. de O.; PEZZOPANE, M.; TAGLIAFERRE, C. Estudo comparativo da estimativa da evapotranspiração 
de referência para três localidades do Estado do Espírito Santo no período seco. Idesia, v.25, n.3, p.75-84, 2007.

TAGLIAFERRE, C.; SILVA, R.; ROCHA, F.; SANTOS, L. Estudo comparativo de diferentes metodologias para determinação da evapotranspiraçao de referência em Eunapolis BA. Revista Caatinga v.23, n.1. p.103-111, 2010.

WILlMOTT, C.J. Some comments on evaluation of model performance, Bulletin of American Meteorological Society, v.63, p.13091313, 1982. 